Supporting Information for:

\title{
Influence of Salt Concentration on Hydrated Polymer Relative Permittivity and State of Water Properties
}

\author{
Kevin Chang, Hongxi Luo, and Geoffrey M. Geise* \\ Department of Chemical Engineering \\ University of Virginia \\ 102 Engineers' Way, P.O. Box 400741 \\ Charlottesville, VA 22904 USA
}

*To whom correspondence should be addressed: geise@virginia.edu (Tel: +1-434-924-6248, Fax: +1-434-982-2658) 
Table S1. Water uptake, $w_{u}$, water volume fraction, $\phi_{w}$, and dry polymer density data measured at $22 \pm 1{ }^{\circ} \mathrm{C}$. Water uptake measurements were made on samples initially equilibrated with either DI water or an aqueous solution of $0.01,0.1$, or $1.0 \mathrm{~mol} / \mathrm{L} \mathrm{NaCl}$. The uncertainty was taken as one standard deviation from the mean of three measurements. Hydrolysis time, z, refers to the length of time (in hours) that the XL-pGMA-z samples were immersed in $0.5 \mathrm{~mol} / \mathrm{L} \mathrm{H}_{2} \mathrm{SO}_{4}$ at $40{ }^{\circ} \mathrm{C}$.

\begin{tabular}{|c|c|c|c|c|c|c|c|c|c|c|}
\hline \multirow[b]{2}{*}{ Polymer } & \multirow[b]{2}{*}{$\begin{array}{l}\text { Hydrolysis } \\
\text { Time, z (h) }\end{array}$} & \multirow[b]{2}{*}{$\begin{array}{c}\text { Dry Density } \\
\left(\mathrm{g} / \mathrm{cm}^{3}\right)\end{array}$} & \multicolumn{2}{|c|}{$\begin{array}{l}\text { Measured using } \\
\text { pure water }\end{array}$} & \multicolumn{2}{|c|}{$\begin{array}{l}\text { Measured using } \\
0.01 \mathrm{~mol} / \mathrm{L} \mathrm{NaCl}\end{array}$} & \multicolumn{2}{|c|}{$\begin{array}{l}\text { Measured using } \\
0.1 \mathrm{~mol} / \mathrm{L} \mathrm{NaCl}\end{array}$} & \multicolumn{2}{|c|}{$\begin{array}{l}\text { Measured using } \\
1.0 \mathrm{~mol} / \mathrm{L} \mathrm{NaCl}\end{array}$} \\
\hline & & & $w_{u}$ & $\phi_{w}$ & $w_{u}$ & $\phi_{w}$ & $w_{u}$ & $\phi_{w}$ & $w_{u}$ & $\phi_{w}$ \\
\hline \multirow{3}{*}{ XL-pGMA-z } & 7 & $1.19 \pm 0.02$ & $\begin{array}{c}0.061 \pm \\
0.012\end{array}$ & $\begin{array}{c}0.068 \pm \\
0.013\end{array}$ & $\begin{array}{c}0.033 \pm \\
0.001\end{array}$ & $\begin{array}{c}0.037 \pm \\
0.001\end{array}$ & $\begin{array}{c}0.030 \pm \\
0.007\end{array}$ & $\begin{array}{c}0.034 \pm \\
0.008\end{array}$ & $\begin{array}{c}0.015 \pm \\
0.002\end{array}$ & $\begin{array}{c}0.018 \pm \\
0.002\end{array}$ \\
\hline & 9 & $1.26 \pm 0.04$ & $0.118 \pm$ & $0.129 \pm$ & $0.059 \pm$ & $0.070 \pm$ & $0.064 \pm$ & $0.075 \pm$ & $0.041 \pm$ & $0.050 \pm$ \\
\hline & 11 & $1.23 \pm 0.03$ & $\begin{array}{c}0.168 \pm \\
0.018\end{array}$ & $\begin{array}{c}0.172 \pm \\
0.019\end{array}$ & $\begin{array}{c}0.087 \pm \\
0.021\end{array}$ & $\begin{array}{c}0.097 \pm \\
0.024\end{array}$ & $\begin{array}{c}0.089 \pm \\
0.009\end{array}$ & $\begin{array}{c}0.099 \pm \\
0.011\end{array}$ & $\begin{array}{c}0.070 \pm \\
0.007\end{array}$ & $\begin{array}{c}0.079 \pm \\
0.008\end{array}$ \\
\hline
\end{tabular}

Table S2. Amounts of strongly-bound, intermediate, and bulk-like water present in XL-pGMA-Z when equilibrated in DI water or 0.01, 0.1 , or $1.0 \mathrm{~mol} / \mathrm{L}$ aqueous $\mathrm{NaCl}$ solutions. The results were obtained by fitting the OD stretch of each FTIR spectrum of XL-pGMA-Z with three Gaussian curves. Each curve represents a different water state, and the area of each Gaussian curve was divided by the total area of the OD stretch. The three values for each material and condition sum to the overall volume fraction of sorbed water, $\phi_{w}$. Hydrolysis time, z, refers to the length of time (in hours) that the XL-pGMA-z samples were immersed in $0.5 \mathrm{~mol} / \mathrm{L} \mathrm{H}_{2} \mathrm{SO}_{4}$ at $40{ }^{\circ} \mathrm{C}$.

\begin{tabular}{|c|c|c|c|c|c|c|c|c|c|c|c|c|c|}
\hline \multirow[b]{2}{*}{ Polymer } & \multirow[b]{2}{*}{$\begin{array}{l}\text { Hydrolysis } \\
\text { Time, z (h) }\end{array}$} & \multicolumn{3}{|c|}{$\begin{array}{l}\text { Measured using } \\
\text { pure water }\end{array}$} & \multicolumn{3}{|c|}{$\begin{array}{l}\text { Measured using } \\
0.01 \mathrm{~mol} / \mathrm{L} \mathrm{NaCl}\end{array}$} & \multicolumn{3}{|c|}{$\begin{array}{l}\text { Measured using } \\
0.1 \mathrm{~mol} / \mathrm{L} \mathrm{NaCl}\end{array}$} & \multicolumn{3}{|c|}{$\begin{array}{l}\text { Measured using } \\
1.0 \mathrm{~mol} / \mathrm{L} \mathrm{NaCl}\end{array}$} \\
\hline & & $\begin{array}{c}\text { Strongly } \\
\text {-bound } \\
\text { water } \\
\left(\times 10^{-2}\right)\end{array}$ & $\begin{array}{c}\text { Inter- } \\
\text { mediate } \\
\text { water } \\
\left(\times 10^{-2}\right)\end{array}$ & $\begin{array}{l}\text { Bulk- } \\
\text { like } \\
\text { water } \\
\left(\times 10^{-2}\right)\end{array}$ & $\begin{array}{c}\text { Strongly } \\
\text {-bound } \\
\text { water } \\
\left(\times 10^{-2}\right)\end{array}$ & $\begin{array}{c}\text { Inter- } \\
\text { mediate } \\
\text { water } \\
\left(\times 10^{-2}\right)\end{array}$ & $\begin{array}{l}\text { Bulk- } \\
\text { like } \\
\text { water } \\
\left(\times 10^{-2}\right)\end{array}$ & $\begin{array}{c}\text { Strongly } \\
\text {-bound } \\
\text { water } \\
\left(\times 10^{-2}\right)\end{array}$ & $\begin{array}{c}\text { Inter- } \\
\text { mediate } \\
\text { water } \\
\left(\times 10^{-2}\right)\end{array}$ & $\begin{array}{l}\text { Bulk- } \\
\text { like } \\
\text { water } \\
\left(\times 10^{-2}\right)\end{array}$ & $\begin{array}{c}\text { Strongly } \\
\text {-bound } \\
\text { water } \\
\left(\times 10^{-2}\right)\end{array}$ & $\begin{array}{c}\text { Inter- } \\
\text { mediate } \\
\text { water } \\
\left(\times 10^{-2}\right)\end{array}$ & $\begin{array}{l}\text { Bulk- } \\
\text { like } \\
\text { water } \\
\left(\times 10^{-2}\right)\end{array}$ \\
\hline \multirow{3}{*}{$\begin{array}{c}\text { XL- } \\
\text { pGMA-z }\end{array}$} & 7 & 0.45 & 6.27 & 0.08 & 0.35 & 3.23 & 0.13 & 0.30 & 2.86 & 0.25 & 0.11 & 1.59 & 0.10 \\
\hline & 9 & 0.82 & 11.33 & 0.75 & 0.58 & 6.00 & 0.41 & 0.68 & 6.43 & 0.39 & 0.30 & 4.39 & 0.31 \\
\hline & 11 & 1.37 & 14.56 & 1.28 & 0.74 & 8.07 & 0.89 & 0.81 & 8.33 & 0.76 & 0.51 & 6.85 & 0.53 \\
\hline
\end{tabular}



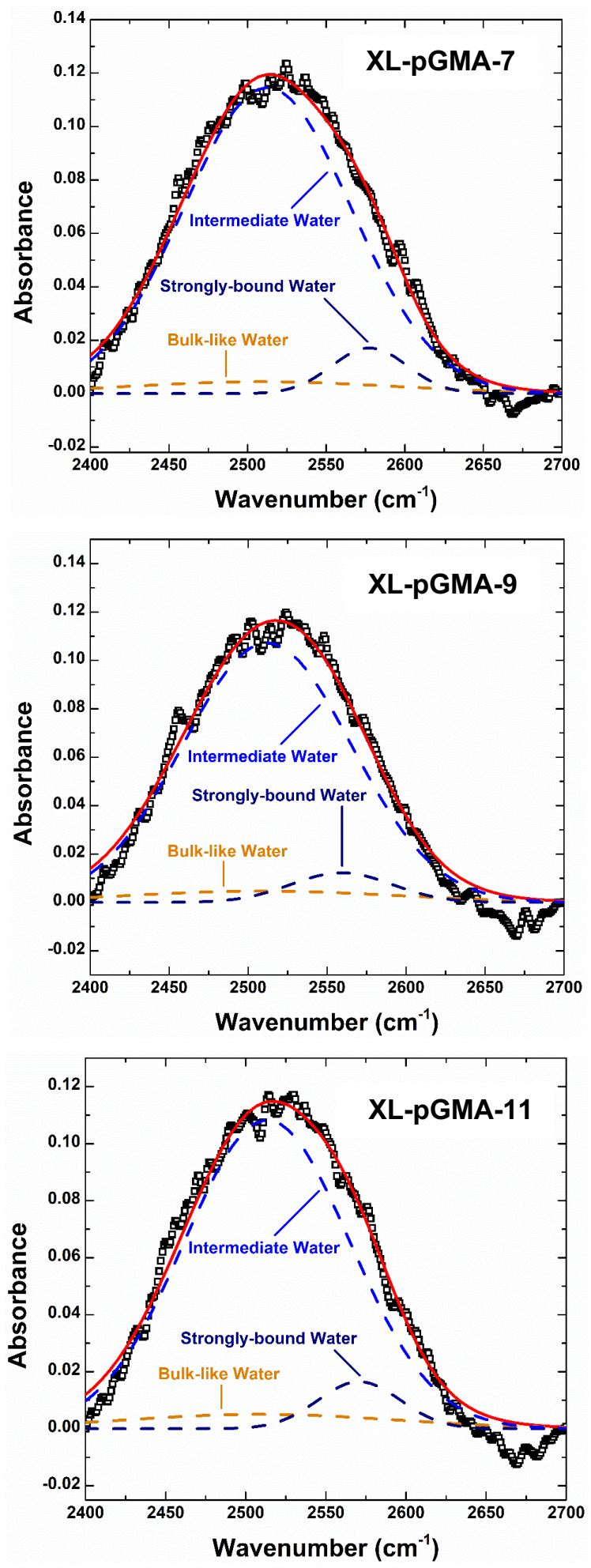

Figure S1. Representative FT-IR spectra for XL-pGMA-7 (top), -9 (middle), and -11 (bottom) materials that were equilibrated in $1.0 \mathrm{~mol} / \mathrm{L} \mathrm{NaCl}$. The three Gaussian peaks that represent the three water states (bulk-like water, intermediate water, and strongly-bound water) are labeled on each figure. 


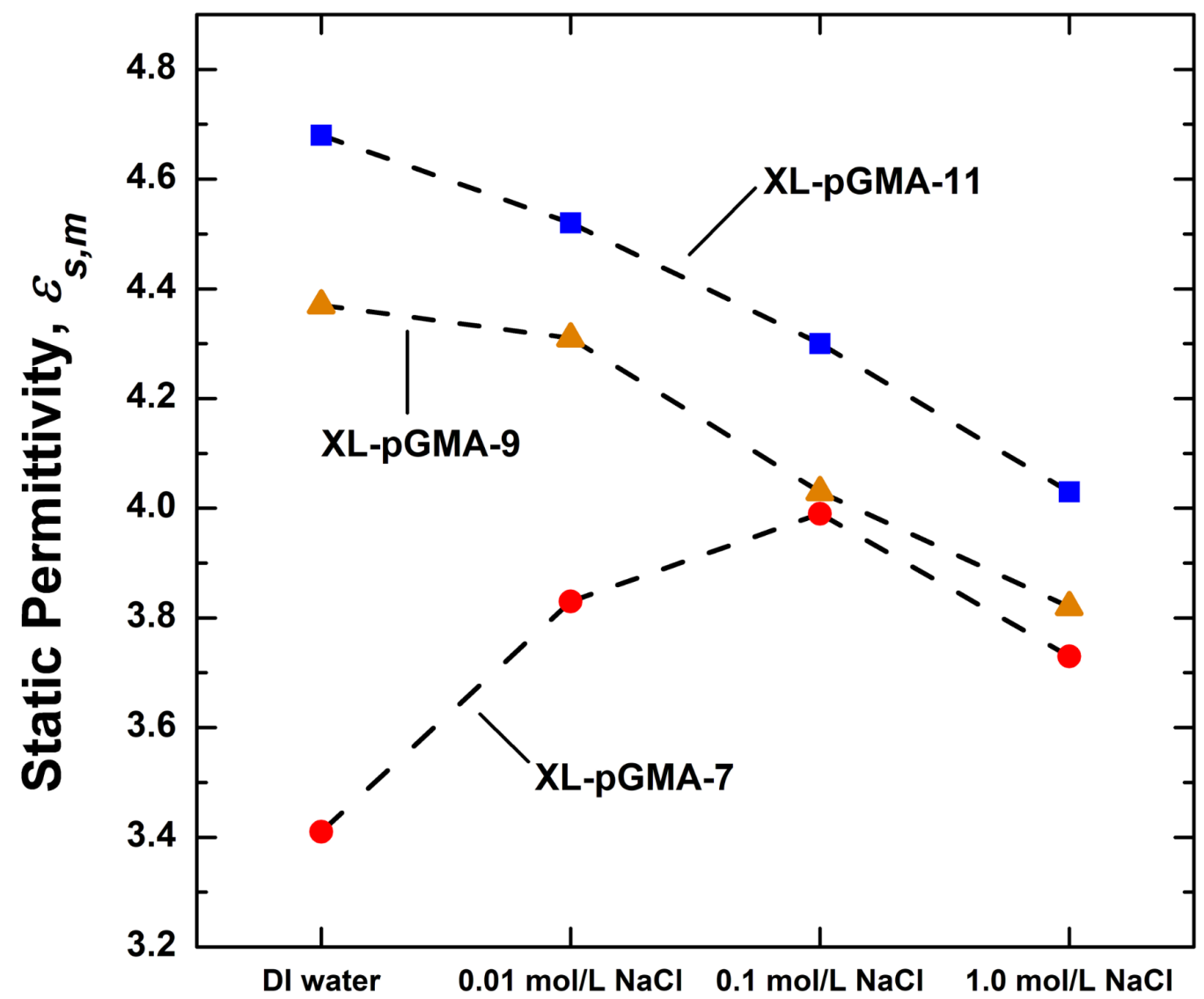

Figure S2. Static permittivity of hydrated XL-pGMA-Z measured using samples that were equilibrated in DI water or $0.01,0.1$, and $1.0 \mathrm{~mol} / \mathrm{L}$ aqueous $\mathrm{NaCl}$ solutions. The dashed line is included to guide the eye. 


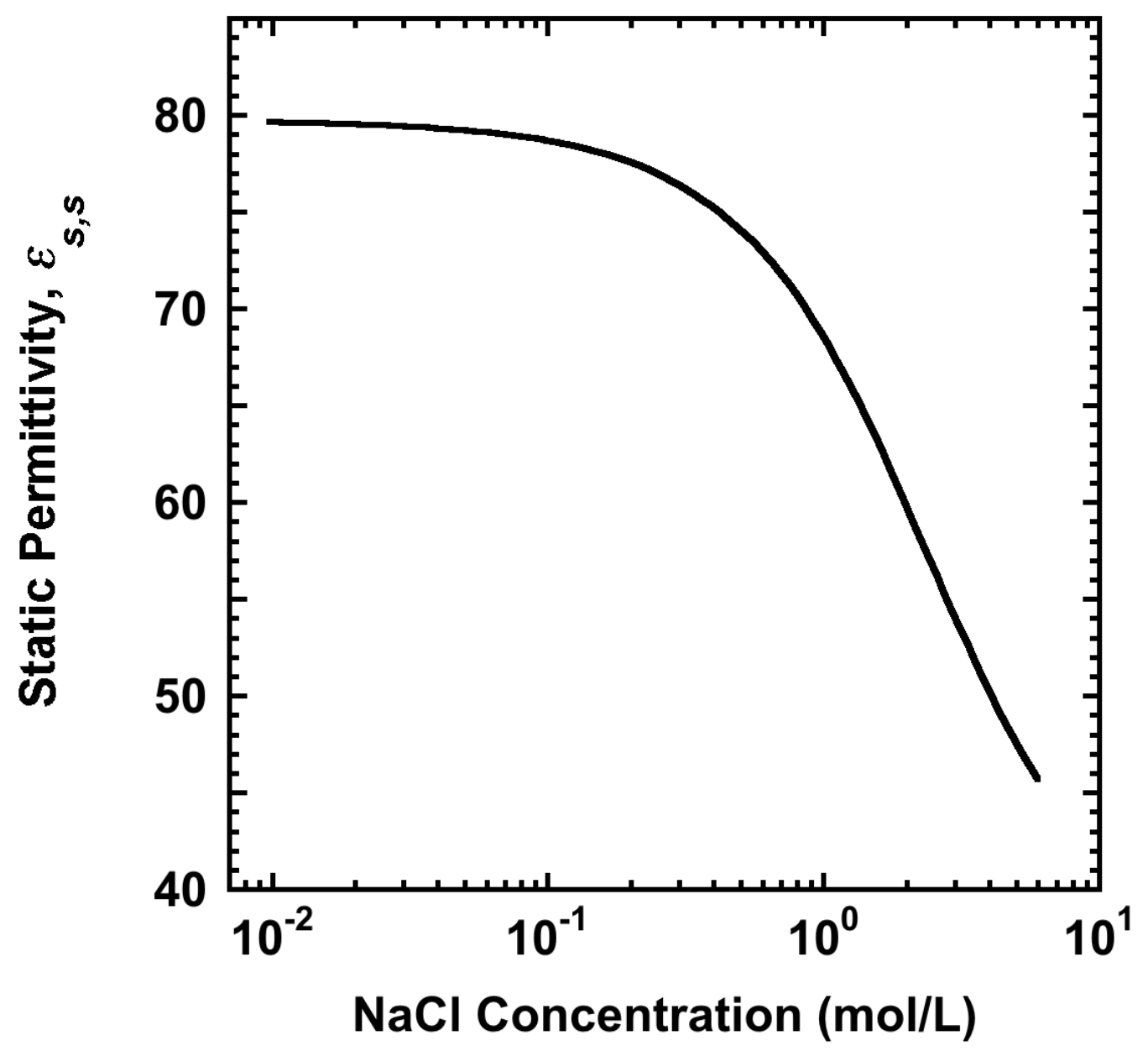

Figure S3. Static permittivity as a function of $\mathrm{NaCl}$ concentration for bulk aqueous $\mathrm{NaCl}$ solution. ${ }^{1}$ Adapted from Hasted, J. B.; Ritson, D. M.; Collie, C. H., Dielectric properties of aqueous ionic solutions. Parts I and II. J. Chem. Phys. 1948, 16, 1-21, with the permission of AIP Publishing.

\section{References}

1. Hasted, J. B.; Ritson, D. M.; Collie, C. H., Dielectric properties of aqueous ionic solutions. Parts I and II. J. Chem. Phys. 1948, 16, 1-21. 